\title{
A computer simulation of solidification taking into account the movement of the liquid phase
}

\author{
Robert Dyja ${ }^{1, *}$, Elżbieta Gawrońska ${ }^{1}$, Andrzej Grosser ${ }^{1}$ \\ ${ }^{1}$ Czestochowa University of Technology, ul. J.H. Dąbrowskiego 69, 42-201 Częstochowa, Poland
}

\begin{abstract}
In the paper, we present the results of solidification simulation taking into account the movement of the liquid phase. The results are obtained from an author software which is implemented on the base of a stabilized finite elements method (Petrov-Galerkin formulation). Using that formulation the Navier-Stokes equation is solved together with the convection term (Boussinesq approximation). The Finite Element Method (FEM) formulation is responsible for solidification, approximating the solution of the heat conduction equation (with the internal heat source term responsible for the heat released during the phase transition). The movement of the liquid phase in a solidifying cast that is caused by convection can significantly affect the process of heat transfer from the casting to the mold, which in turn has an influence on the temperature distribution in the cast and may cause a change in the location of the defects. The presented results allow to assess under what conditions the effect of convection on the solidification process is significant.
\end{abstract}

Keywords: solidification, Navier-Stokes, Streamline Upwind Petrov-Galerkin, Pressure Stabilized Petrov-Galerkin

\section{Introduction}

Solidification is still one of the most difficult process for modelling. Many physical processes that takes place from micro scale up to macro adds present significant difficulties, when someone tries to prepare suitable computational model. During years, many researchers came with models, that could give reasonable results [1,2].

However, many models had some simplifications and limits. Example of such limit was omitting forces of convection in liquid metal. This can lead to significant differences between temperature map from simulation and temperatures observed in real casting. Additional problem is that without convection it is impossible to model such things like distribution of species in casting or solidification shrinkage, which are important factors of castings quality [3].

However, increasing complexity of models, present problem in their implementation. Nowadays, complex models must be implemented with use of technologies like parallel computers [4], accelerated architectures like GPU [5], or using special organization of calculations [6].

\footnotetext{
* Corresponding author: robert.dyja@icis.pcz.pl

Reviewers: Juraj Grenčík, Kateryna Kravchenko
} 


\section{Mathematical model}

The governing equation for modelling solidification process is based on heat transfer equation with source term:

$$
\rho c \dot{T}+\rho c(\mathbf{u} \cdot \nabla) T=\lambda \nabla^{2} T+\dot{q},
$$

where $\rho$ is density $\left[\mathrm{kg} / \mathrm{m}^{3}\right], c$ is specific heat $[\mathrm{J} /(\mathrm{kg} \mathrm{K})], T$ is temperature $[\mathrm{K}], \mathbf{u}$ is velocity from convection force $[\mathrm{m} / \mathrm{s}], \lambda$ is thermal conductivity $[\mathrm{W} /(\mathrm{m} \mathrm{K})]$ and $q$ is heat source connected with heat of solidification $\left[\mathrm{J} / \mathrm{m}^{3}\right]$.

Boundary conditions, that are used in model with equation (1) are the Newton boundary condition on external sides of mold and contact condition for heat exchange between casting and mold.

With use of the apparent heat capacity formulation it is possible to obtain following form of equation (1):

$$
c^{*} \dot{T}+\rho c(\mathbf{u} \cdot \nabla) T=\lambda \nabla^{2} T,
$$

where $c^{*}$ is the approximation of the effective heat capacity. There are various methods for obtaining this approximation. Here, the Morgan method is used:

$$
c^{*}=\frac{H^{t}-H^{t-1}}{T^{t}-T^{t-1}},
$$

where $H$ is enthalpy [J] and $t$ in upper script is time level.

Liquid metal in this model is assumed to be Newtonian fluid. This allows to write Navier-Stokes set of equations as:

$$
\begin{gathered}
\rho \dot{\mathbf{u}}+\rho((\mathbf{u} \cdot \nabla) \mathbf{u})-\nabla p+\rho \mu\left((\nabla \mathbf{u})+(\nabla \mathbf{u})^{T}\right)+\rho \mu \frac{f_{l}}{K_{\epsilon}} \mathbf{u}=\rho \mathbf{f} \\
\nabla \cdot \mathbf{u}=0
\end{gathered}
$$

where $\mathrm{p}$ is pressure [Pa], $\mu$ is viscosity $[\mathrm{kg} /(\mathrm{m} \mathrm{s})], f_{l}$ is liquid fraction [-] and $K_{\varepsilon}$ is the permeability of the mushy zone $[\mathrm{m}]$.

This equation needs to be accompanied by appropriate set of initial and boundary conditions. Initial $\boldsymbol{u}$ is set as initial condition and between mold and casting the no-slip condition is used.

The right hand side part of equation (4) describes body forces that arose in liquid. The first part is connected with buoyance force that is approximated by Boussinesq formula:

$$
\mathbf{f}=-\beta \mathbf{g}\left(T-T_{0}\right),
$$

where $\beta$ is expansion coefficient $[1 / \mathrm{K}], \boldsymbol{g}$ is gravitational acceleration $\left[\mathrm{m} / \mathrm{s}^{2}\right], T_{0}$ is reference temperature $[\mathrm{K}]$, which in this case was temperature from initial conditions.

The last part of left hand side of equation (4) is drag force that appears in mushy zone from interaction between liquid and already solidified metal. This model assumes that solidified part is immovable [7]. The permeability of the mushy zone is approximated by the Kozeny-Carman equation:

$$
K_{\varepsilon}=K_{0} \frac{f_{l}^{3}}{\left(1-f_{l}\right)^{2}}
$$

where $K_{0}$ is secondary dendrite arm spacing [m] and $f_{l}$ is liquid fraction [-].

Liquid fraction and solid fraction are connected with simple relation:

$$
f_{l}=1-f_{s}
$$


where value of $f_{s}$ is taken from phase equilibrium graph relationship:

$$
f_{S}=\frac{1}{1-k} \frac{T_{L}-T}{T_{M}-T_{L}}
$$

where $T_{L}$ is liquidus temperature $[\mathrm{K}], T_{M}$ is solidification temperature of pure component $[\mathrm{K}]$ and $k$ is solute partition coefficient $[-]$.

Equations (1), (4) and (5) are discretized using Finite Element Method, which leads to following set of equations:

$$
\begin{gathered}
\mathbf{M}^{S} \mathbf{T}+\left(\mathbf{N}^{S} \mathbf{T}+\mathbf{K}^{S}\right) \mathbf{T}=0 \\
\mathbf{M u}+(\mathbf{N}+\mathbf{K}) \mathbf{u}-\mathbf{G} \mathbf{p}+\mathbf{D} \mathbf{u}=\mathbf{F} \\
\mathbf{G}^{T} \mathbf{u}=0
\end{gathered}
$$

where superscript $S$ is used to set apart matrices used in solidification equation and $\mathbf{T}, \mathbf{u}$, $\mathbf{p}$ are vectors of unknown temperature, velocity and pressure. Elements of matrices $\mathbf{M}^{s}, \mathbf{M}$, $\mathbf{N}^{s}, \mathbf{N}, \mathbf{K}^{s}, \mathbf{K}, \mathbf{G}^{T}, \mathbf{G}$ and $\mathbf{D}$ can be calculated from the following formulas:

$$
\begin{gathered}
M_{\alpha \beta}^{S}=\int_{\Omega} c^{*} S_{a} S_{b} \mathrm{~d} \Omega \\
N_{\alpha \beta}^{S}=\int_{\Omega} \rho c S_{a} u_{k} S_{b, k} \mathrm{~d} \Omega \\
K_{\alpha \beta}^{S}=\int_{\Omega} \lambda S_{a, j} S_{b, j} \mathrm{~d} \Omega \\
M_{\alpha \beta}=\int_{\Omega} \rho c S_{a} S_{b} \mathrm{~d} \Omega \\
K_{\alpha \beta}=\int_{\Omega} \mu S_{a, k} S_{b, k} \delta_{i j} \mathrm{~d} \Omega+\int_{\Omega} \mu S_{a, j} S_{b, i} \mathrm{~d} \Omega \\
N_{\alpha \beta}=\int_{\Omega} \rho S_{a} u_{k} S_{b, k} \delta_{i j} \mathrm{~d} \Omega \\
G_{\alpha}=\int_{\Omega} S_{a, i} S_{b} \mathrm{~d} \Omega \\
D_{\alpha \beta}=\int_{\Omega} \rho \mu \frac{f_{l}}{K_{\epsilon}} S_{a} S_{b} \delta_{i j} \mathrm{~d} \Omega \\
G_{\beta}^{T}=\int_{\Omega} S_{b, j} S_{a} \mathrm{~d} \Omega \\
F_{\alpha}=\int_{\Omega} \rho S_{a} f_{i} \mathrm{~d} \Omega,
\end{gathered}
$$

where $\alpha, \beta$ are indices of single element matrix, $a$ and $b$ have range up to number of nodes in element, $i, j, k$ have range up to number of dimensions, $S$ are shape functions of finite elements and $\delta$ is Kronecker delta.

Equations in this form can be solved with carefully selected finite elements [8]. In this work, approach that uses stabilized Finite Element is used [9], which allows to avoid limits imposed by the Ladyzhenskaya-Babuska-Breezi condition.

Special care is needed for treating drag force part in stabilization [10]. This work uses approach, that determines values of stabilization coefficient not only by velocity of liquid, but also limits them proportionally to volume of liquid fraction.

In calculations, it is assumed that small time step is used, hence in determination of actual values of material properties, when temperature was needed, temperature from 
previous time step was used. This strategy allows to treat solidification equation as linear and even solve it independently from Navier-Stokes equations, which gives better overall performance [11]. Moreover, such approach makes possible to use lumped mass matrix in solidification equation $[11,12]$.

With those assumptions in mind and using theta scheme for time integration, the final form of equations solved in the model is:

$$
\begin{gathered}
{\left[\mathbf{M}^{s}+\mathbf{M}_{S U P G}^{S}+\Delta t \theta\left(\mathbf{N}^{s}+\mathbf{N}_{S U P G}^{s}+\mathbf{K}^{s}\right)\right] \mathbf{T}^{t+1}=} \\
=\left[\mathbf{M}^{s}+\mathbf{M}_{S U P G}^{s}+\Delta t(1-\theta)\left(\mathbf{N}^{s}+\mathbf{N}_{S U P G}^{s}+\mathbf{K}^{s}\right)\right] \mathbf{T}^{t} \\
{\left[\mathbf{M}+\mathbf{M}_{S U P G}+\Delta t \theta\left(\mathbf{N}+\mathbf{N}_{S U P G}+\mathbf{K}+\mathbf{D}+\mathbf{D}_{S U P G}\right)\right] \mathbf{u}^{t+1}-\Delta t \mathbf{G} \mathbf{p}^{t+1}+} \\
+\left[\mathbf{M}+\mathbf{M}_{S U P G}+\Delta t(1-\theta)\left(\mathbf{N}+\mathbf{N}_{S U P G}+\mathbf{K}+\mathbf{D}+\mathbf{D}_{S U P G}\right)\right] \mathbf{u}^{t}= \\
=\Delta t\left[\theta\left(\mathbf{F}+\mathbf{F}_{S U P G}\right)+(1-\theta)\left(\mathbf{F}+\mathbf{F}_{S U P G}\right)\right] \\
{\left[\mathbf{M}_{P S P G}+\Delta t \theta\left(\mathbf{G}^{T}+\mathbf{N}_{P S P G}+\mathbf{D}_{P S P G}\right)\right] \mathbf{u}^{t+1}-\Delta t \mathbf{G}_{P S P G} \mathbf{p}^{t+1}+} \\
+\left[\mathbf{M}_{P S P G}+\Delta t(1-\theta)\left(\mathbf{G}^{T}+\mathbf{N}_{P S P G}+\mathbf{D}_{P S P G}\right)\right] \mathbf{u}^{t}= \\
=\Delta t\left[\theta \mathbf{F}_{P S P G}+(1-\theta) \mathbf{F}_{P S P G}\right]
\end{gathered}
$$

where matrices with $S U P G$ and $P S P G$ are terms supplied by stabilization, $\Delta t$ is time step and $\theta$ is parameter determining type of time integration scheme ( 0 for Euler Backward and 0.5 for Cranck-Nicolson).

This model uses Newton-Raphson approach for linearization of Navier-Stokes equations.

\section{Simulations}

Model described in section 2 was implemented as an in-house $\mathrm{C}++$ program. This program uses TalyFEM [13] and PETSc [14] libraries. TalyFEM library implements finite element formulas like calculations of shape functions or Gauss quadrature. PETSc library is used for linear algebra operations.

The results for two cases are presented. All simulations use linear triangle finite elements. First is small casting (square shape with 0.05 [m] side length), the second is larger one $(0.5[\mathrm{~m}]$ side length), see Fig. 1 . Those two cases allow to asses importance of convection in solidification.

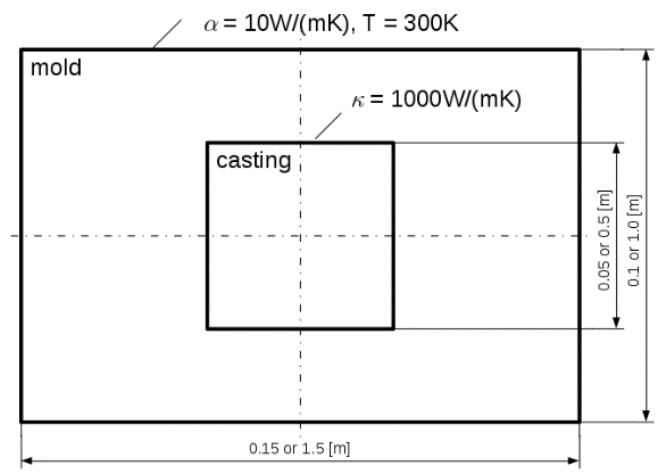

Fig. 1. View of domain shape used in simulations 
The boundary conditions utilized following parameters: Newton boundary condition on all sides of mold had the environment temperature equal to $300[\mathrm{~K}]$ and heat exchange coefficient was equal to $10[\mathrm{~W} /(\mathrm{m} \mathrm{K})]$. Continuity condition assumed value of $1000[\mathrm{~W} /(\mathrm{m} \mathrm{K})]$ for heat transfer through separation layer.

The material properties are summarised in Table 1 (for casting) and Table 2 (for mold).

Table 1. Material properties for casting

\begin{tabular}{|l|l|l|}
\hline \multicolumn{1}{|c|}{ Quantity name } & Unit & Value \\
\hline Density $\rho_{s}$ & $\mathrm{~kg} / \mathrm{m}^{3}$ & 2824 \\
\hline Density $\rho_{l}$ & $\mathrm{~kg} / \mathrm{m}^{3}$ & 2498 \\
\hline Specific heat $c_{s}$ & $\mathrm{~J} /(\mathrm{kg} \mathrm{K})$ & 1077 \\
\hline Specific heat $c_{l}$ & $\mathrm{~J} /(\mathrm{kg} \mathrm{K})$ & 1275 \\
\hline Thermal conductivity $\lambda_{s}$ & $\mathrm{~W} /(\mathrm{m} \mathrm{K})$ & 262 \\
\hline Thermal conductivity $\lambda_{l}$ & $\mathrm{~W} /(\mathrm{m} \mathrm{K})$ & 104 \\
\hline Solidus temperature $T_{s}$ & $\mathrm{~K}$ & 853 \\
\hline Liquidus temperature $T_{l}$ & $\mathrm{~K}$ & 926 \\
\hline $\begin{array}{l}\text { Solidification temperature of } \mathrm{pure} \\
\text { component } T_{M}\end{array}$ & $\mathrm{~K}$ & 933 \\
\hline Eutectic temperature $T_{E}$ & $\mathrm{~K}$ & 821 \\
\hline Heat of solidification $L$ & $\mathrm{~J} / \mathrm{kg}$ & 390000 \\
\hline Solute partition coefficient $k$ & - & 0.125 \\
\hline Viscosity $\mu$ & $\mathrm{kg} /(\mathrm{m} \mathrm{s})$ & 0.004 \\
\hline Expansion coefficient $\beta$ & $1 / \mathrm{K}$ & 0.0001 \\
\hline Secondary dendrite arm spacing $K_{0}$ & $\mathrm{~m}$ & $1.4 * 10^{-11}$ \\
\hline
\end{tabular}

Table 2. Material properties for mold

\begin{tabular}{|l|l|l|}
\hline Quantity name & Unit & Value \\
\hline Density $\rho_{s}$ & $\mathrm{~kg} / \mathrm{m}^{3}$ & 7500 \\
\hline Specific heat $c_{s}$ & $\mathrm{~J} /(\mathrm{kg} \mathrm{K})$ & 620 \\
\hline Thermal conductivity $\lambda_{s}$ & $\mathrm{~W} /(\mathrm{m} \mathrm{K})$ & 40 \\
\hline
\end{tabular}

The first series of results present temperature map in four time moments (12.5 [s], $25.0[\mathrm{~s}], 37.5[\mathrm{~s}], 50.0[\mathrm{~s}])$ for small square. It is presented in Fig. 2. The effect of convection is only slightly visible in the beginning of simulation. With increasing time, effects of convection are less visible. However, in time moment 50 [s], location of hot spot can be viewed as minimally moved toward top. In this case symmetry of results is easily observable.

Corresponding pressure and velocity map is presented in Fig. 3. The pressure is presented as colour map, while velocity is presented as black vectors. Their size and density corresponds to the magnitude of velocity. The main observation here is that even in places with mushy zone there are no visible fluctuations of pressure, which shows effectiveness of used stabilization technique. After 25 [s] there is almost no liquid phase movement.

In larger case the effect of convection is better visible. It can be observed as fluctuations of temperatures presented in Fig. 4. In Fig. 5, we can see that during whole simulation time, liquid metal had nonzero velocity. 


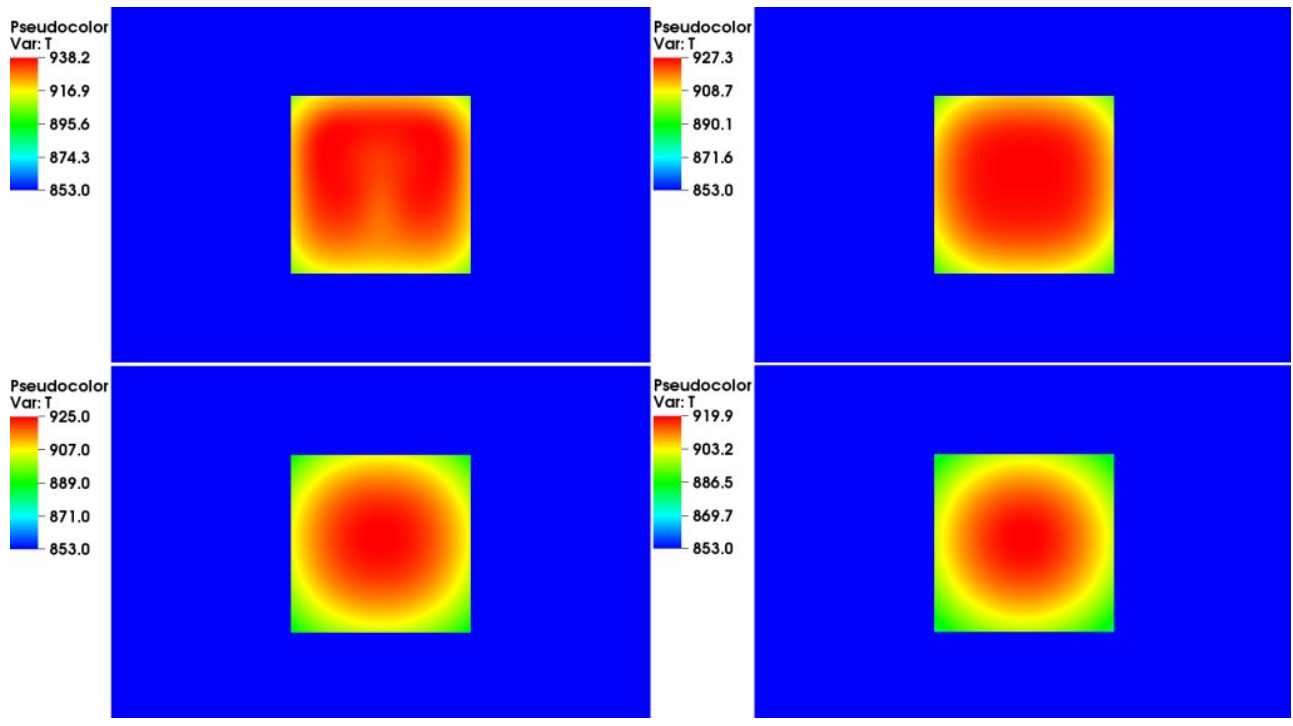

Fig. 2. Temperature in different time moments $(12.5[\mathrm{~s}], 25.0[\mathrm{~s}], 37.5[\mathrm{~s}], 50.0[\mathrm{~s}]$ - starting from upper left corner). Size of single side of casting is $0.05[\mathrm{~m}]$

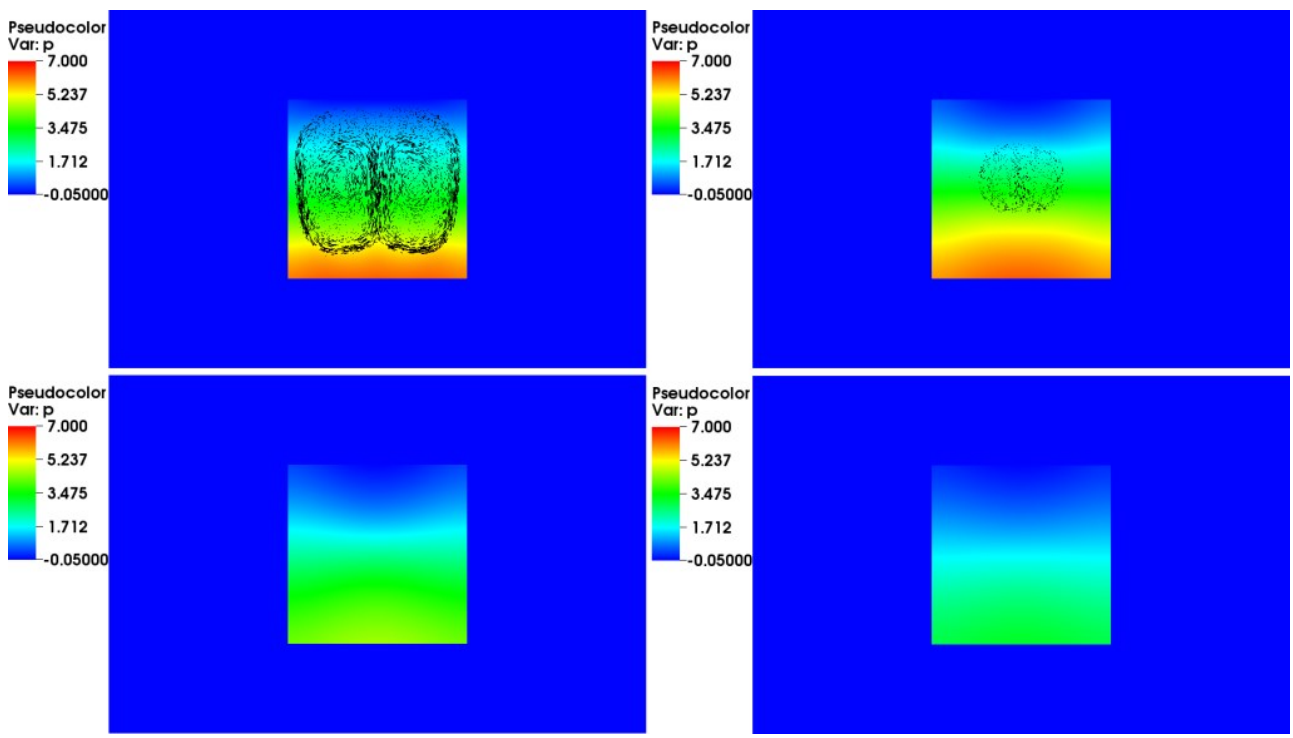

Fig. 3. Pressure with velocity vectors in different time moments $(12.5[\mathrm{~s}], 25.0[\mathrm{~s}], 37.5[\mathrm{~s}], 50.0[\mathrm{~s}]$ starting from upper left corner). Size of single side of casting is $0.05[\mathrm{~m}]$

More detailed comparison can be done with help of plots presented in Fig. 6 and Fig. 7, where also results from case without convection are used. Fig. 6 presents cooling curves taken in different places of casting (in centre, top, bottom and close to right wall). When there is no convection, curves from bottom, top and right spot are the same. However, with convection, it can be easily seen, that bottom cools faster then top spot.

Fig. 7 shows results of the same analysis, but for the large case. Here differences are even more visible. Without convection, the results from places close to walls are the same. 


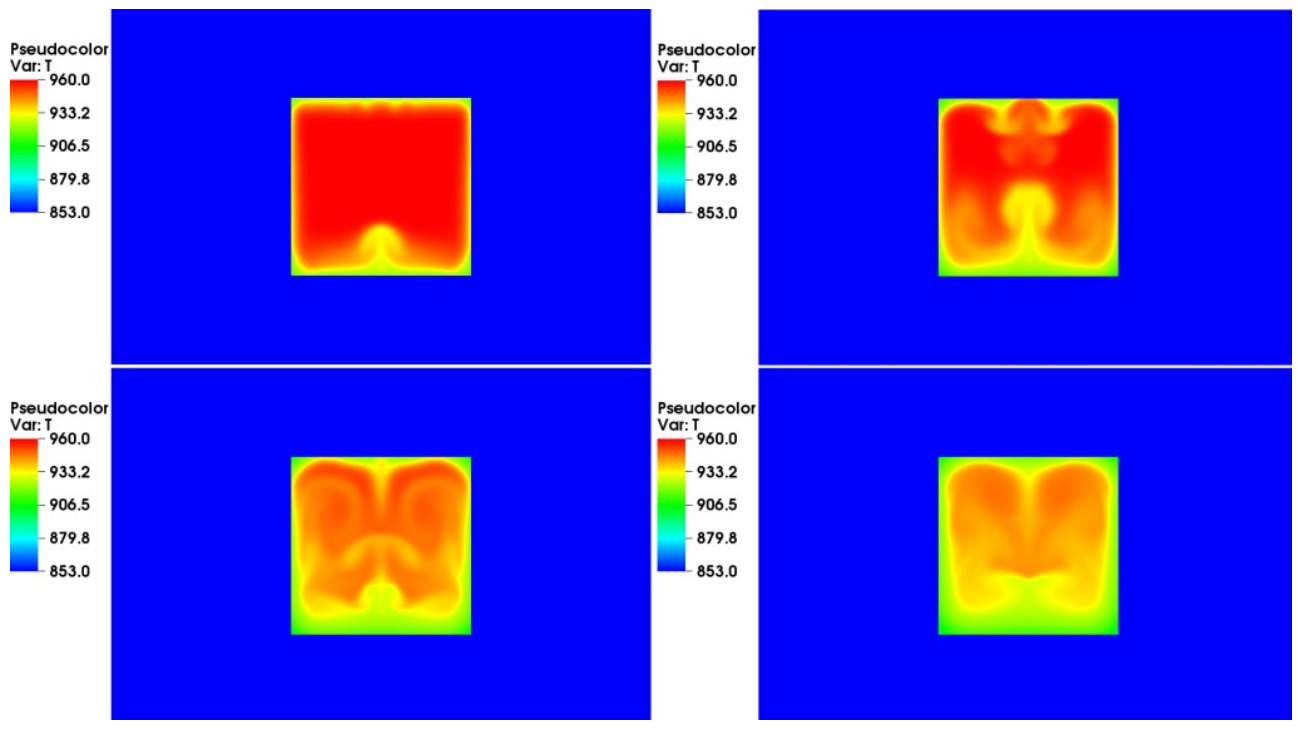

Fig. 4. Temperature in different time moments $(12.5[\mathrm{~s}], 25.0[\mathrm{~s}], 37.5[\mathrm{~s}], 50.0[\mathrm{~s}]$ - starting from upper left corner). Size of single side of casting is $0.5[\mathrm{~m}]$

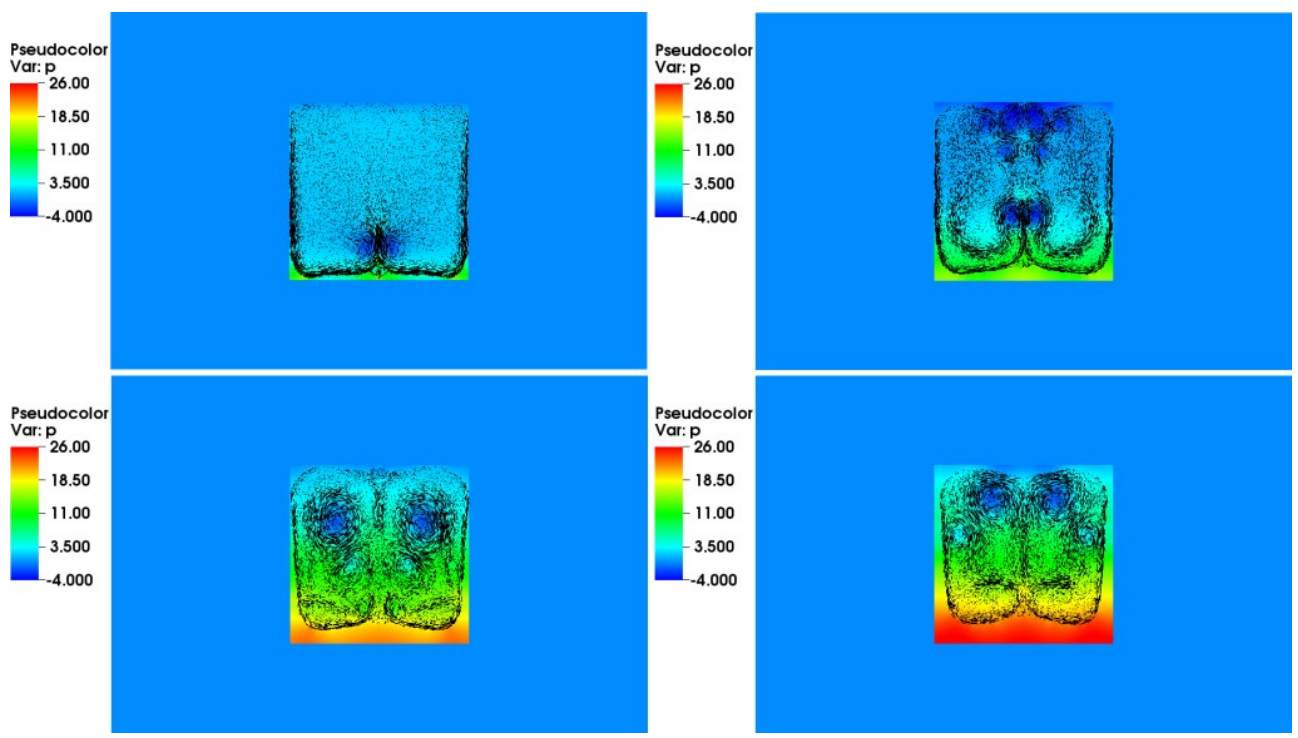

Fig. 5. Pressure with velocity vectors in different time moments $(12.5[\mathrm{~s}], 25.0[\mathrm{~s}], 37.5[\mathrm{~s}], 50.0[\mathrm{~s}]$ starting from upper left corner). Size of single side of casting is $0.5[\mathrm{~m}]$ 


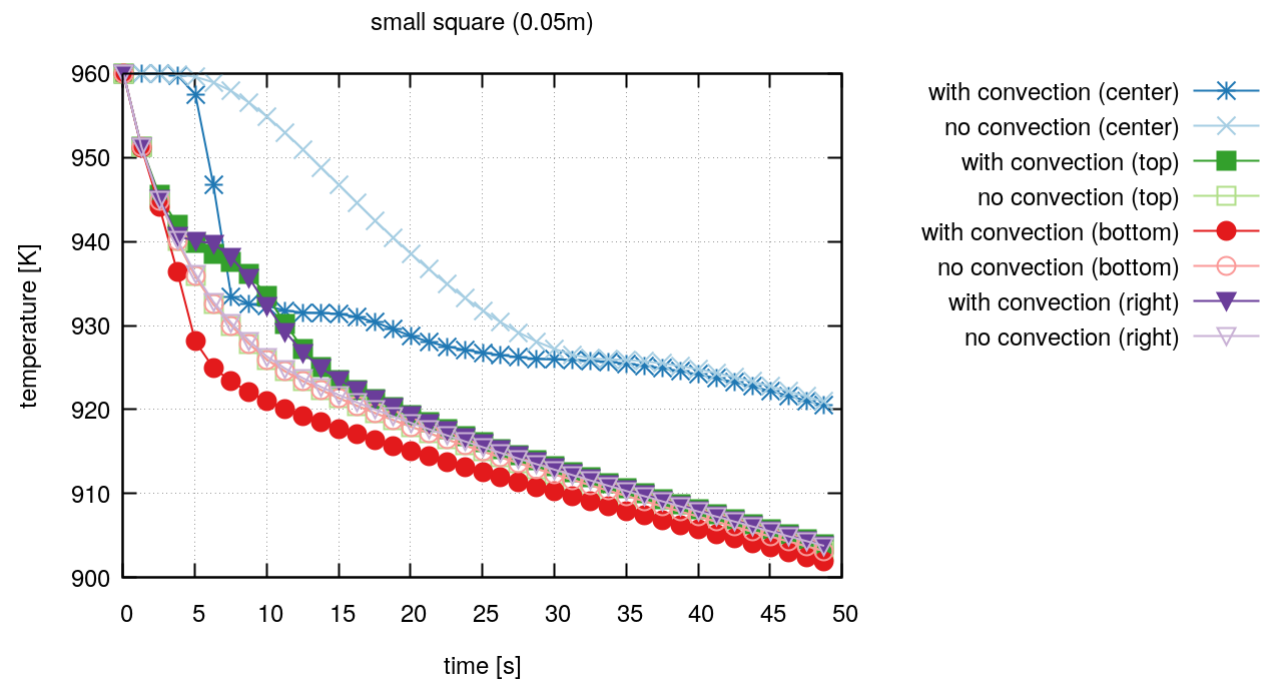

Fig. 6. Cooling curves for the small case obtained in different places of casting. As a comparison, cooling curves, for cases without convection are also plotted
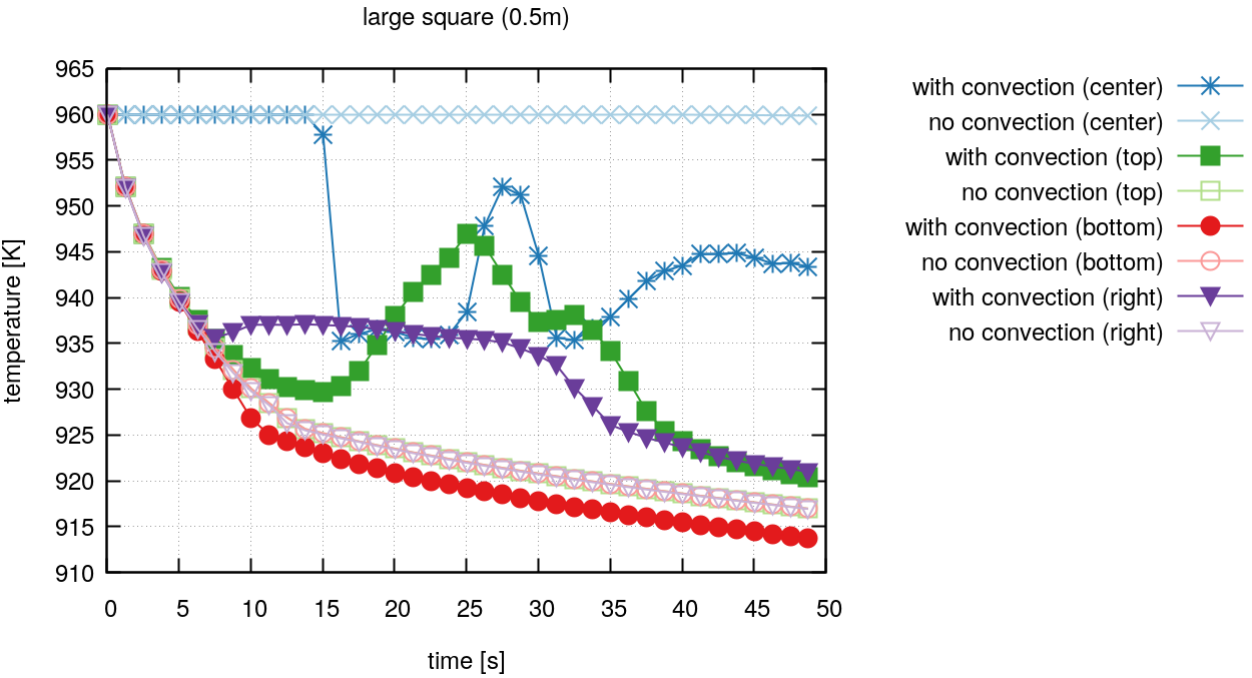

Fig. 7. Cooling curves for the large case obtained in different places of casting. As a comparison, cooling curves, for cases without convection are also plotted

\section{Conclusion}

Presented work tackles with problem of convection in solidification simulations. It presents development of model and difficulties linked with presence of momentum and continuity equations. It shows study of conditions when convection can be a significant factor. It also presents comparison with model that does not take into account this phenomena. Presented results shows that even for small casting convection can affect results in comparison with immovable liquid state. 
Plans for future work include experimental comparison of results, because presented model was only checked against benchmark problems so far.

\section{References}

1. A. Bokota, S. Iskierka, Finite element method for solving diffusion-convections problems in the presence of a moving heat point source. Finit. Elem. Anal. Des. 17 (2), 89-99 (1994)

2. T. Skrzypczak, E. Wegrzyn-Skrzypczak, J. Winczek, Effect of natural convection on directional solidification of pure metal. Arch. Metal. Mater. 60 (2), 835-841 (2015)

3. D. M. Stefanescu, Science and Engineering of Casting Solidification. (New York, Kluwer Academic, 2002)

4. W. Feng. Q. Xu, B. Liu, Microstructure simulation of alluminium alloy using parallel computng technique. ISIJ International 42 (7), $702-707$ (2002)

5. G. Michalski, N. Sczygiol, Using CUDA architecture for the computer simulation of the casting solidification process. Hong Kong: Lecture Notes in Engineering and Computer Science, 933-937 (2014)

6. E. Gawronska, N. Sczygiol, Application of mixed time partitioning methods to raise the efficiency of solidification modelling. 12th International Symposium on Symbolic and Numeric Algorithms (SYNASC), 99-103 (2010)

7. W. D. Bennon, F. P. Incropera, A continuum model for momentum, heat and species transport in binary solid-liquid phase change systems - I. Model formulation. Int. J. Heat Mass Transf. 30 (10), 2161-2170 (1987)

8. F. Brezzi, On the existence, uniquess and approximation of saddle-point problems arising from lagrangian multipliers. Esaim Math. Model. Numer. Anal. 8 (R2), 129151 (1974)

9. A. N. Brooks, T. J. R. Hughes, Streamline Upwind/Petrov-Galerkin formulations for convection dominated flows with particular emphasis on the incompressible NavierStokes equations. Computer Methods in Applied Mechanics and Engineering - Special Edition on the 20th Anniversary, 199-259 (1990)

10. N. Zabaras, D. Samanta, A stabilized volume-averaging finite element method for flow in porous media and binary alloy solidification processes. Int. J. Numer. Methods Eng. 60 (5), 1-38 (2004)

11. R. Dyja, E. Gawronska, A. Grosser, Numerical problems related to solving the NavierStokes equations in connection with the heat transfer with the use of FEM. Procedia Eng. 177, 78-85 (2017)

12. M. Zych, Effect of mass matrix forms on numerical simulation results in heat conduction modeling. J. Appl. Math. Comput. Mech. 14 (3), 149-156 (2015)

13. H. K. Kodali, B. Ganapathysubramanian, A computational framework to investigate charge transport in heterogeneous organic photovoltaic devices. Comput. Methods Appl. Mech. Eng. 247, 113-129 (2012)

14. S. Balay, W. D. Gropp, L. C. McInnes, B. F. Smith, Efficient Management of Parallelism in Object Oriented Numerical Software Libraries. Modern Software Tools in Scientific Computing, 163-202 (1997) 